\title{
Critical properties of the XXZ model with long-range interactions on the double chain
}

\author{
J. P. de Lima ${ }^{\mathrm{a}, *}$, L. L. Gonçalves ${ }^{\mathrm{b}}$ \\ ${ }^{a}$ Departamento de F'isica, Universidade Federal do Piauí, Campus Min. Petrônio Portela, 64049-550 Teresina, Piauí, Brazil \\ ${ }^{\mathrm{b}}$ Departamento de Engenharia Metalúrgica e de Materiais, Universidade Federal do Ceará, Campus do Pici, 60455-760 Fortaleza, Ceará, Brazil
}

Received 12 June 2005; revised 13 June 2005; accepted 14 June 2005

\begin{abstract}
'The $X X Z$ model $(s=1 / 2)$ in a transverse field on a double chain with a uniform long-range interaction among the $z$ components of the spins is considered. The nearest-neighbour interactions are restricted to the components in the $x y$ plane and to the spins within the same chain leg, ' such that the Hamiltonian is given by $H=-\sum_{m=1}^{2} J_{m} \sum_{j=1}^{N}\left(S_{m, j}^{x} S_{m, j+1}^{x}+S_{m, j}^{y} S_{m, j+1}^{y}\right)-\frac{I}{N} \sum_{m, n=1}^{2} \sum_{j, k=1}^{N} S_{m, j}^{z} S_{n, k}^{z}-h \sum_{m=1}^{2} \sum_{j=1}^{N} S_{m, j}^{z}$, where $N$ is the number of sites of the lattice and $m, n(m, n=1,2)$ label the chain legs. The model is solved exactly by introducing the Jordan-Wigner ' and integral Gaussian transformations, which map the Hamiltonian in a non-interacting fermion system and corresponds to an extension of 'the model recently studied by the authors for a single chain. The equation of state is obtained in closed form, and the critical classical (at , $T>0$ ) and quantum (at $T=0$ ) behaviours can be determined exactly. The quantum critical surface is determined in the space generated by the transverse field and interaction parameters, and the crossover lines separating the different critical regimes are also obtained. It is also shown that, differently from the results obtained for the single chain, the system can present multiple quantum transitions.
\end{abstract}

(c) 2018 Elsevier B.V. All rights reserved.

PACS: $05.50 .+\mathrm{q} ; 64.60 .-\mathrm{i} ; 64.60 . \mathrm{Kw}$

Keywords: Quantum transitions; $X Y Z$ model; Double-chain; Long-range interactions

The critical behaviour of the XXZ model with long-range interactions among the spins along the $\mathrm{z}$ direction, in a single chain, has been recently considered by the authors [1]. In that paper they present a comprehensive study of the quantum and classical transitions undergone by the model. Although the model presents first- and second- order quantum and classical transitions, which is a very important feature, no multiple transitions are present. However, multiple quantum transitions can occur in magnetic systems as it has been shown in the experimental results recently obtained for $\mathrm{ZrZn}_{2}$ [2]. This result has been the main motivation for studying spin models which are exactly soluble and can present multiple quantum transitions. Therefore we will consider the XXZ model $(s=1 / 2)$ in a transverse field on a double chain with long-range uniform interaction among $\mathrm{z}$ components of spins. The nearestneighbour interactions are assumed isotropic, and restricted to the spin components in the XY plane and to the spins within the same chain leg. It should be noted that due to the isotropy of the nearest-neighbour interactions the total magnetization along the $z$ direction is conserved. Assuming periodic boundary

\footnotetext{
* Corresponding author.

Email address: pimentel@ufpi.br (J. P. de Lima).
}

conditions the Hamiltonian is given by

$$
\begin{gathered}
H=-\sum_{m=1}^{2} J_{m} \sum_{j=1}^{N}\left(S_{m, j}^{x} S_{m, j+1}^{x}+S_{m, j}^{y} S_{m, j+1}^{y}\right) \\
-\sum_{m, n=1}^{2} \frac{I}{N} \sum_{j, k=1}^{N} S_{m, j}^{z} S_{n, k}^{z}-h \sum_{m=1}^{2} \sum_{j=1}^{N} S_{m, j}^{z}
\end{gathered}
$$

where $N$ is the number of sites of one leg and $m(m=1,2)$ labels the chain legs. By introducing the Jordan-Wigner transformation [3] and a Gaussian transformation, we can write the partition function as

$$
\begin{aligned}
Z_{N}= & \left(\frac{N}{2 \pi}\right)^{1 / 2} \int_{-\infty}^{\infty} d x \exp \left[-\frac{N}{2} x^{2}\right] \times \operatorname{Tr} \exp [-\bar{H}] \times \\
& \exp \left[-N \sum(\beta h+\sqrt{2 \beta I})\right]
\end{aligned}
$$

where $\beta=1 / k_{B} T$, the effective Hamiltonian $\bar{H}$ is given by

$\bar{H} \equiv \sum_{m, j} \beta J_{m}\left(c_{m, j}^{\dagger} c_{m, j+1}+c_{m, j+1}^{\dagger} c_{m, j}\right)+\sum_{m, j}(\beta h+\sqrt{2 \beta I}) c_{m, j}^{\dagger} c_{m, j}$,

and it has been used the fact that the long-range interaction commutes with $H$. Then, introducing the spatial Fourier transform, the Hamiltonian $\bar{H}$, apart from a constant, can be written 
in diagonal form with energy excitations $\bar{E}_{k_{m}}=\beta J_{m} \cos \left(k_{m}\right)+$ $\beta h+\sqrt{2 \beta I} x$. Then the partition function can be recast in the form

$$
Z_{N}=\left(\frac{N}{2 \pi}\right)^{1 / 2} \int_{-\infty}^{\infty} d x \exp \left[-\frac{N}{2} x^{2}\right] \prod_{m, k}\left[1+\exp \left(-\bar{E}_{k_{m}}\right)\right]
$$

and, in the thermodynamic limit, can be evaluated by the saddlepoint method. Following this procedure, the free energy functional can be written in terms of the magnetization, $M^{z}$, and at $T=0$, is given by

$f=h+4 I M^{z}\left(M^{z}+1\right)-\frac{1}{\pi}\left(J_{1} \sin \varphi_{1}+J_{2} \sin \varphi_{2}\right)-\frac{1}{\pi}\left(\varphi_{1}+\varphi_{2}\right)$,

where $M^{z}$ is explicitly given by

$$
M^{z}=\frac{\varphi_{1}+\varphi_{2}}{2 \pi}-\frac{1}{2}
$$

with $\varphi_{m}=\arccos \left(-\left(h+4 I M^{z}\right) / J_{m}\right)$. It should be noted that for $J_{1}=J_{2}$ the results obtained so far reproduce the known ones for the single chain [1]. By minimizing the free energy functional, given in eqs (5) and (6), and the by using stability condition

$$
\frac{\partial^{2} f}{\partial\left(M^{z}\right)^{2}} \geq 0
$$

we can determine the equation of state and obtain the phase diagram at $T=0$. Whithout loss of generality, we will restrict the analysis of the phase diagram to the region $J_{2} / J_{1} \geqslant 1$, which presents the main features of the model. In order to charaterize the different quantum phases displayed by the model we present in Fig. 1 the magnetization as a function of the field for different values of the long-range interaction. As it can be seen, for $I \leq$ 0 , the transitions are of second order since the magnetization is continuous at the transition points. On the hand, for $I>0$ the magnetization is discontinuous at the the transition points which is a signature of a first order transition.

The quantum critical surface obtained is shown in Fig. 2 where, as already mentioned, we have quantum phase transitions of first and second order. In the region $I \leq 0$, it can be shown that there are two critical surfaces of second order quantum phase transition. These critical surfaces are given explicitly by the expressions $h=J_{1}-2 I \arccos \left(-J_{1} / J_{2}\right) / \pi$, which corresponds to the magnetization $M^{z}=1 / 2 \pi \arccos \left(-J_{1} / J_{2}\right)$, and by $h=J_{2}-2 I$, which corresponds to the magnetization $M^{z}=1 / 2$. These two surfaces intercept when $J_{1}=J_{2}$ as it can be seen in the diagram shown in Fig.2. For $I>0$ the model presents three lines corresponding to the quantum phase transitions of first order, with a triple line which is also shown in Fig. 2. In this case, it is not possible to obtain an explicit equation for the critical surface and it has been determined numerically.

The interception of the critical surfaces of first and second orders occurs at $I=0$, and corresponds to a bicritical line since it contains the known bicritical point present for $J_{1}=J_{2}[1,4]$, when the results reduce to the ones for the single chain.

Moreover, it should be noted that, for a given set of parameters, the system presents multiple transitions as a function of the field, as it can be seen in Fig. 2. In the second-order region, the multipe transitions can also be obtained from the explicit equation for the critical surface given above.

Finally, we would like to point out that, for $J_{1}=J_{2}$, the quantum critical behaviour is identical to the one obtained for the model when we have a single chain $[1,4]$, and as already mentioned we do not have multiple transitions.

This work was partially financed by the Brazilian agencies CNPq and Capes. The authors would like to thank Dr. A. P. Vieira for a critical reading of the manuscript.

\section{References}

[1] L. L. Gonçalves, L. P. S. Coutinho and J. P. de Lima, Physica A 345 (2005) 71.

[2] M. Uhlarz, C. P. Pfleiderer and S. M. Hayden, Phys. Rev. Lett. 93(2004) 256404.

[3] P. Jordan, E. Wigner, Z. Physik 47 (1928) 631.

[4] L. L. Gonçalves, A. P. Vieira and L. P. S. Coutinho, J. Magn. Magn. Mater.226-230(2001) 613.

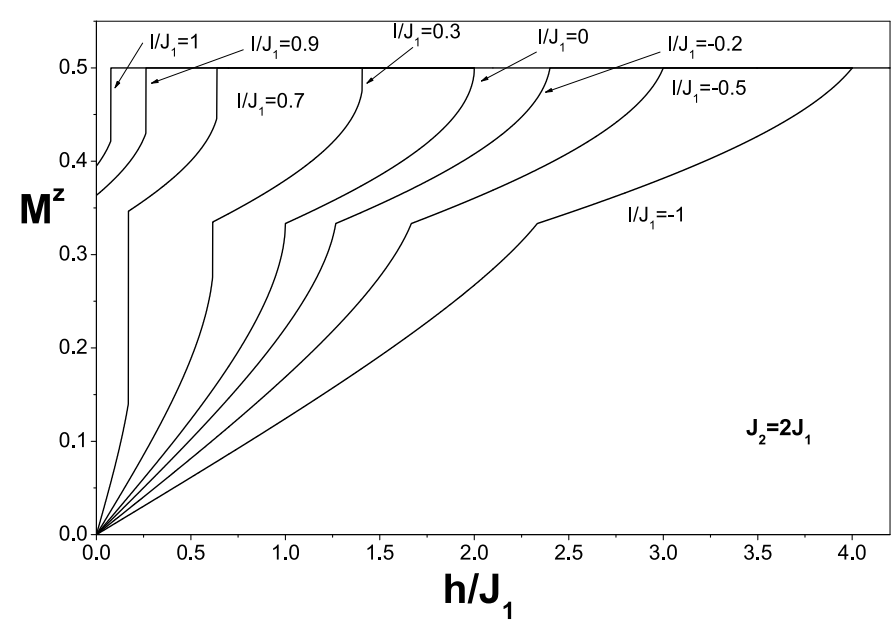

Fig. 1. Magnetization (at $T=0$ ) as a function of the field for different values of the long-range interactions.

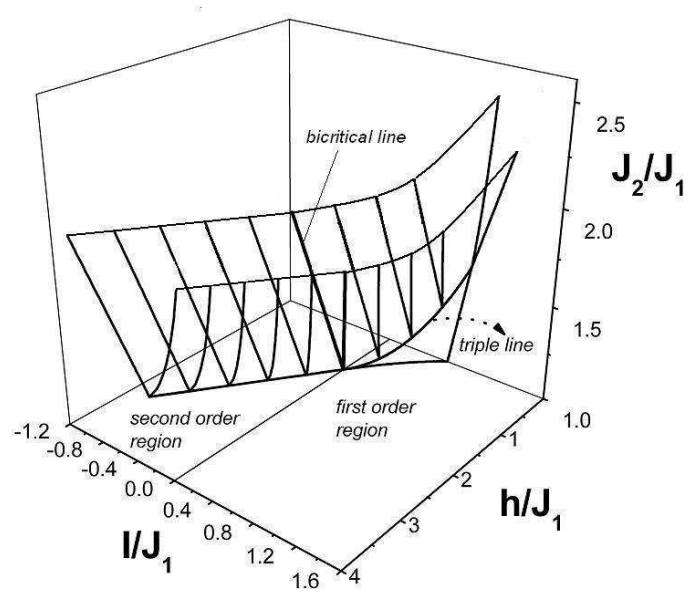

Fig. 2. Phase diagram of the model at $T=0$ and $J_{2} / J_{1}>1$. 